\title{
Gait training On Spastic Diplegic children-A physiotherapy Approach
}

\author{
Deivendran Kalirathinam, Jeganathan Arumugam \\ ${ }^{1}$ Deivendran Kalirathinam, Associate Professor, MAEER'SPhysiotherapy College MIMER Medical College \& \\ Hospital, Talegaon Dhabade, Pune, Maharastra, India . \\ ${ }^{2} J$ geganathan arumugam, Associate Professor, MAEER'SPhysiotherapy College MIMER Medical College \& \\ Hospital, Talegaon Dhabade, Pune, Maharastra, India .
}

\begin{abstract}
Background: Cerebral palsy is defined as a non-progressive insult to a developing or immature central nervous system (CNS), particularly to those areas that affect motor function. People with spastic diplegia typically walk slowly and have difficulty in performing activities such as walking up and down steps or running. People with spastic diplegia typically walk slowly and have difficulty in performing activities such as walking up and down steps or running. Purpose of the study: The aim of this study is to find out that whether quadriceps strengthening can improve the gait and if this exercise protocol is successful then it can be implemented for children with spastic diplegia. Method: 30 Spastic diplegic children of both genders with age 4-12 years satisfying inclusion criteria were included for the study. The children were divided in two groups Group A( Experimental Group) and Group B (Control Group) having 15 children each. Cadence and distance covered in 3 min walk test was calculated before the test and was documented as Pre test Score.This assessment was done by making the child to walk in room with a length of 10 meter. Two bright coloured poles were kept at the end of 8 meters and the child was asked to walk around it.The researcher assessed initially the cadence Then a rest period of 10 minutes was given and 3 minute walk test was performed. Time was calculated by stop watch. The interventions were given for 5 days a week for 2 weeks and each session lasted for 90 minutes. Group A was given Stretching exercises, quadriceps strengthening exercises with free ankle weights half squats, sit to stand and step ups. Group B was administered only conventional exercises. Post treatment data was taken and statistical analysis was performed. Results: The results of unpaired t-test comparing the difference of pre and post treatment values of Group A and Group B showed improvement in mean difference in values of cadence $(t=12.2, \quad p=0.000)$ and distance ambulated in 3 Minute Walk Test $(t=14.627$, $p=0.000)$.Conclusion: The results of this randomized clinical trial support the view that two weeks of strengthening program was effective in increasing cadence and distance walked in three min walk test in children with spastic diplegia.
\end{abstract}

Keywords: Cereberal Palsy, Quadriceps Strengthening, Gait, 3 Minute Walk Test

\section{Back Ground:}

Cerebral palsy is defined as a non-progressive insult to a developing or immature central nervous system (CNS), particularly to those areas that affect motor function. The impairment of voluntary motor control is the hallmark of cerebral palsy (CP). Spastic diplegia, the most prevalent form of cerebral palsy, is characterized by motor incoordination, primarily in the lower extremities, that impairs many functional abilities, most notably ambulation. ${ }^{4}$ It results from brain damage at birth that prevents proper development of the pyramidal tract, meaning that certain nerve receptors in the spine are in turn unable to properly absorb the gamma amino butyric acid which would otherwise properly regulate tone in the affected areas.

People with spastic diplegia typically walk slowly and have difficulty in performing activities such as walking up and down steps or running. The most common gait patterns are characterized by excessive knee and hip flexion, implicating weakness of the ankle plantar flexors, knee extensors, and hip extensors. ${ }^{14}$ Spasticity in the legs is rarely so great as to totally prevent ambulation-most people with spastic diplegia can walk - but regardless, spastic diplega does result in the signature "scissor gait". The gait of a person with spastic diplegia cerebral palsy is typically characterized by a crouched gait

The ability to walk is a major concern of the parents of children with $\mathrm{CP}$, and improving or maintaining this ability is often considered to be the primary focus of most therapeutic interventions addressing the motor problems seen in children with spastic diplegia. Quadriceps weakness diminishes knee control and deficient in stance are most pronounced. External forces tend to hyperflex the knee and a variety of compensations are employed to preserve stability in weight bearing. It is possible to increase muscle strength in these children through traditional strength training programs. The Quadriceps femoris strength training program in absence of knee flexion contracture would produce improvement in gait performance by reducing the degree of knee crouch during stance. 
People with spastic diplegia typically walk slowly and have difficulty in performing activities such as walking up and down steps or running. The most common gait patterns are characterized by excessive knee and hip flexion, implicating weakness of the ankle plantar flexors, knee extensors, and hip extensors. ${ }^{14}$ Spasticity in the legs is rarely so great as to totally prevent ambulation-most people with spastic diplegia can walk - but regardless, spastic diplega does result in the signature "scissor gait". Researchers determined the effects of progressive resisted muscle strengthening for children with a diagnosis of cerebral palsy. The literature suggests that progressive resisted muscle exercises of an isolated muscle group increases muscle performance in children and adults with cerebral palsy, with no adverse effects on muscle spasticity ${ }^{7}$. The gait of a person with spastic diplegia cerebral palsy is typically characterized by a crouched gait. Dave Smith et al (2004) $)^{8}$, advocated that those interested in improving their muscular size, strength, power and/or endurance should perform one set of each exercise to muscular failure Toe walking and flexed knees are common attributes and can be corrected with proper treatment and gait analysis. Although the majority of children with spastic diplegia are eventually able to ambulate, the acquisition of this skill is delayed and differs qualitatively from normal pediatric gait. Damiano DL et al $(1995)^{5}$, conducted a study to determine the effect of quadriceps strengthening in attempt to decrease crouch during gait in children with spastic diplegia and found effective.

An increasing number of studies have reported that strength-training programmes can improve strength. Damiano et al (1995 \& 1998) conducted a study on two different isotonic training programs, one for the knee extensors alone and one for multiple lower extremity muscles, depending on individual areas of weakness, including hip flexors, extensors, and adductors, knee extensors, and ankle dorsiflexors and plantar flexors, each documenting improvement in strength and gait parameters. Blundell SW et al $(2003)^{2}$,determined the effects of intensive task specific strength training on lower limb strength and functional performance in children with cerebral palsy. Damiano DL et al $(2002)^{6}$, conducted an annotation to summarize existing research on strength testing and training primarily focusing on cerebral palsy.

In another study, according to Damiano et al, hamstring strength was measured before and after a quadriceps strengthening program in children with $\mathrm{CP}$ to determine whether the program caused an inadvertent increase in strength in the spastic muscle due to abnormal cocontraction or stretch responses elicited in the antagonist during agonist strengthening. Researchers determined the reliability and magnitude of error of three lower limb clinical measures for children with cerebral palsy concluded that these tools should be used with caution when evaluating changes in young children with cerebral palsy ${ }^{1}$.The quadriceps showed a mean strength increase of more than $50 \%$ with no significant change in the hamstring values.

The aim of this study is to find out that whether quadriceps strengthening can improve the gait and if this exercise protocol is successful then it can be implemented for children with spastic diplegia.The objective of this study is to examine the effect of dynamic quadriceps strength training on cadence and distance ambulated during a 3 min walk test.

\section{Methodology}

30 Spastic diplegic children of both genders with age 4-12 years who were able to ambulate for 3 minutes on a level surface with or without assistive device, who were able to follow multi step commands, who have Grade 3 or more than Grade 3 in voluntary control grading were selected. Children who had recent history of botux injections, dorsal rhizotomy or use of baclofen pump,cardiopulmonary problems, auditory and visual problems were excluded from the study. After obtaining the informed consent from the caretakers, the children were divided in two groups Group A( Experimental Group) and Group B (Control Group) having 15 children each. Cadence and distance covered in 3 min walk test was calculated before the test and was documented as Pre test Score.

This assessment was done by making the child to walk in room with a length of 10 meter. Two bright coloured poles were kept at the end of 8 meters and the child was asked to walk around it.The researcher assessed initially the cadence. Then a rest period of 10 minutes was given and 3 minute walk test was performed. Time was calculated by stop watch. Pre test data was taken before the starting of the research.The interventions were given for 5 days a week for 2 weeks and each session lasted for 90 minutes. Group A was given Stretching exercises, quadriceps strengthening exercises with free ankle weights half squats, sit to stand and step ups. Group B was administered only conventional exercises.Post treatment data was taken and statistical analysis was performed. Data were analyzed by using student $t$ test with spss 16.0 version for windows 
III. Data Analysis And Results:

TABLE - 1 MEAN, STD.DEV , SEM OF CADENCE

\begin{tabular}{|l|l|l|l|l|l|}
\hline GROUP & & $\mathrm{N}$ & MEAN & STD.DEV & SEM \\
\hline GROUP A & PRE & 15 & 85.21 & 18.1 & 4.837 \\
& POST & 15 & 100.4 & 16.77 & 4.483 \\
\hline GROUP B & PRE & 15 & 73.08 & 23.56 & 6.534 \\
& POST & 15 & 73.92 & 24.19 & 6.71 \\
\hline
\end{tabular}

In GROUP A, 95\% confidence interval for difference: -17.39 to $-12.89, \mathrm{t}=14.527$ with 13 degrees of freedom. $\mathrm{p}=0.000$, The mean difference $=15.19 \mathrm{steps} / \mathrm{min}$

In GROUP B, 95\% confidence interval for difference: -1.859 to $0.1664, t=1.821$ with 12 degrees of freedom. $p$ $=0.094$, The mean difference $=0.84 \mathrm{steps} / \mathrm{min}$

TABLE-2 MEAN, STD.DEV, SEM OF 3 MIN WALK TEST

\begin{tabular}{|l|l|l|l|l|l|}
\hline GROUP & & $\mathrm{N}$ & MEAN & STD.DEV & SEM \\
\hline GROUP A & PRE & 15 & 40 & 13.45 & 3.593 \\
& POST & 15 & 52.36 & 13.68 & 3.656 \\
\hline GROUP B & PRE & 15 & 31.11 & 16.7 & 4.463 \\
& POST & 15 & 31.66 & 17.56 & 4.693 \\
\hline
\end{tabular}

In GROUP A, 95\% confidence interval for difference:- 13.67 to $-11.04, t=20.334$ with 13 degrees of freedom. $p=0.000$, The mean difference $=12.36 \mathrm{~m}$

In GROUP B, 95\% of confidence interval for difference:-1.595 to $-0.4806, t=1.160$ with 12 degrees of freedom. $\mathrm{p}=0.267$, The mean difference $=0.55 \mathrm{~m}$

TABLE-3 Difference in Cadence between both the groups

\begin{tabular}{|l|l|l|l|l|}
\hline GROUP & $\mathrm{N}$ & MEAN(CM) & STD.DEV & SEM \\
\hline GROUP A & 15 & 15.14 & 3.9 & 1.042 \\
\hline GROUP B & 15 & 0.8462 & 1.676 & 0.4647 \\
\hline
\end{tabular}

95\% confidence interval for difference: 11.88 to $16.71 \mathrm{t}=12.20$ with 25 degrees of freedom. $\mathrm{p}=0.000$.The mean difference $=14.29 \mathrm{steps} / \mathrm{m}$

TABLE-4 Difference in 3 Min Walk Test between both the groups

\begin{tabular}{|l|l|l|l|l|}
\hline GROUP & $\mathrm{N}$ & MEAN(CM) & STD.DEV & SEM \\
\hline GROUP A & 14 & 12.36 & 2.274 & 0.607 \\
\hline GROUP B & 13 & 0.6 & 1.863 & 0.516 \\
\hline
\end{tabular}

95\% confidence interval for difference: 10.1 to $13.41 \mathrm{t}=14.62$ with 25 degrees of freedom. $\mathrm{p}=0.000$.The mean difference $=11.76 \mathrm{~m}$

\section{Discussion}

Cerebral palsy is the most common cause of physical disability affecting children. It results in an upper motor neuron syndrome characterized by positive features such as spasticity, co-contraction, and clonus. Eileen G Fowler et al $(2001)^{10}$, conducted a study to test the premise that the performance of exercises with maximum efforts will increase spasticity in people with cerebral palsy and concluded that results do not support the premise that exercise with maximum efforts increase spasticity in people with cerebral palsy. There are also negative features, such as weakness, loss of selective motor control, and balance deficits, that are probably relevant to locomotor prognosis and function. Galea et al $(2005)^{11}$, conducted a study to compare eight lower limb measurements of sagittal plane passive range of motion in 22 children with spastic diplegia. The data confirmed that children with mild spastic diplegia had some restriction in passive range of motion compared to controls. Dodd KJ et al $(2002)^{9}$, determined whether strength training is beneficial for people with cerebralpalsy suggest that training can increase strength and may improve motor activity in people with cerebral palsy without adverse effects. The trials suggest that training can increase strength and may improve motor activity in people with cerebral palsy without adverse effects

This randomized clinical trial provides important new evidence that two week quadriceps strength training can improve the gait in children with spastic diplegia. The results of this study demonstrate significant changes in cadence and distance ambulated in 3 Minute Walk Test. C Andersson et al (2003) ${ }^{3}$, evaluated the effects of a progressive strength training programme on walking ability in adults with cerebral palsy Their findings suggested that a 10 week strength training programme improves muscle strength and walking 
ability without increasing spasticity. Maltais, Desiree et $\mathrm{al}(2005)^{15}$, evaluated the relationship between habitual physical activity and biomechanical treadmill economy in children with cerebal palsyThey concluded that those with high biomechanical treadmill walking economy are the more habitually physically active.

Overall improved trunk and lower extremity strength may have resulted in increased core stability and balance during gait, allowing increased comfort during walking and therefore increased speed. Jan F Morton et al $(2005)^{12}$, investigated the effects of progressive resistance training of quadriceps femoris and hamstrings muscles in children with cerebral palsy. Results included increase in muscle strength, with quadriceps to hamstrings strength changes were retained at follow up. Walking speed and speed rate were increased. The results of this study indicate an increase in distance transversed during a 3 Minute Walk Test. This increase in distance may also correlate with the significant increases in gait velocity, step length and cadence. There may also have been increased stance stability and/or improved muscle health secondary to increased movement allowing them to adapt better to the demands of walking.

Johanne et al $(2002)^{13}$, conducted a study to determine the feasibility of intermittent intensive therapy in severely impaired children with cerebral palsy and to measure the changes in gross motor function,that intermittent program led to improvements in motor function that were maintained over rest periods. Karen $\mathbf{J}$ Dodd et al $(2003)^{14}$, performed a study to evaluate the effects of a home based, six week strength training programme on lower limb strength and physical activity of 21 young people The study concluded that home based training programme can lead to lasting changes in strength of lower limb muscles. Marianne Unger et al $(2006)^{16}$, evaluated the impact of an eight week strength training program targeting multiple muscle groups on gait and perceptions of body image and functional competence. The study concluded that participation in school based strength training can lead to improved degree of crouch gait and improve perception of body image.

It is possible that participation in a strength training programme might lead to more permanent changes in everyday physical activities that in turn might lead to maintenance of strength and activity benefits. Few participants reported that after completing the strengthening programme they could more easily walk up and down the steps at school. These personal achievements suggest that relatively short strengthening programmes can lead to meaningful changes in the lives of people with cerebral palsy and their families.

Optimal management of people with cerebral palsy may include strengthening in combination with other orthopaedic strategies. Functional limitations in people with cerebral palsy can be due to a range of factors such as spasticity and fixed musculoskeletal deformities. In this context, strength training might be viewed as a part of a comprehensive management plan rather than as an isolated intervention.

\section{Conclusion}

The results of this randomized clinical trial support the view that two weeks of strengthening program was effective in increasing cadence and distance walked in three min walk test in children with spastic diplegia. In a complex multifaceted disorder such as cerebral palsy most likely requires a multifaceted approach to rehabilitation. Optimal management may require strengthening in combination with more traditional strategies such as reduction of spasticity, surgical correction of deformities, and orthotic management.

\section{References:}

[1] Adrienne L Fosang et al. Measures of muscle and joint performance in the lower limb of children with cerebral palsy. Developmental Medicine \& Child Neurology 2003, 45:664-670.

[2] Blundell SW et al. Functional strength training in cerebral palsy: a pilot study of a group circuit training class for children aged 4-8 years. Clin Rehabil. 2003 Feb;17(1):48-57.

[3] CAndersson et al. Adults with cerebral palsy: walking ability after progressive strength training. Developmental Medicine and Child Neurology 2003, 45:220-228.

[4] DL Damiano et al. Effects of quadriceps femoris muscle strengthening on crouch gait in children with spastic diplegia. Phys Ther Vol. 75, No. 8, August 1995, pp. 658-667.

[5] Damiano, D.L et al.. Muscle response to heavy resistance exercise in children with spastic cerebral palsy. NCPAD journal vol 37,1995,pp. 731-739.

[6] Diane L Damiano et al. Should we be testing and training muscle strength in cerebral palsy? Developmental Medicine \& Child Neurology 2002, 44: 68-72.

[7] Darrah J et al. Review of the effects of progressive resisted muscle strengthening in children with cerebral palsy: a clinical consensus exercise. Pediatric Physical Therapy, 1997; 9 (1): 12-17.

[8] Dave Smith and Stewart Bruce - Low.Strength training methods and the work of Arthur Jones.Journal of Exercise Physiology,vol 7, no. 6,Dec 2004.

[9] Dodd KJ et al. A systematic review of the effectiveness of strength-training programs for people with cerebral palsy. Arch Phys Med Rehabil. 2002 Aug;83(8):1157-64.

[10] Eileen G Fowler et al. The Effect of Quadriceps Femoris Muscle Strengthening Exercises on Spasticity in Children With Cerebral Palsy. Phys Ther Vol.81,No.6,June2001, pp.1215-1223.

[11] Gaela Kilgour et al. Range of Motion in Children with Spastic Diplegia, GMFCS I-II Compared to Age and Gender Matched Controls. Physical and Occupational Therapy in Pediatrics, 2005, vol 25, issue 3. 
[12] Jan F Morton et al. The effects of progressive resistancetraining for children with cerebral palsy. Clinical Rehabilitation, Vol. 19, No. 3, 283-289 (2005).

[13] Johanne Trahan and Francine Malouin. Intermittent intensive physiotherapy in children with cerebral palsy: a pilot study. Developmental Medicine \& Child Neurology 2002, 44: 233-239 233.

[14] Karen J Dodd et al. A randomized clinical trial of strength training in young people with cerebral palsy. Developmental Medicine \& Child Neurology 2003,45:652-657.

[15] Maltais,Desiree B et al. Habitual physical Activity levels are associated with biomechanical walking economy in children with cerebral palsy.American Journal of Physical Medicine and Rehabilitation. 84(1):36-45,Jan 2005.

[16] Marjan Blackburn et al. Reliability of measurements obtained with Modified Ashworth Scale in the lower extremities of people with stroke. Phys Ther, Vol 82, No.1, January 2002, pp. 25-34.

[17] Marianne Unger et al. Strength training in adolescent learners with cerebral palsy: a randomized controlled trial. Clinical Rehabilitation 2006; 20: 469-477. 